\title{
Níveis Elevados de Netrina-1 e IL-1 $1 \beta$ em Mulheres Idosas com SCA: Pior Prognóstico no Acompanhamento de Dois Anos
}

\author{
High Serum Netrin-1 and IL-1ß in Elderly Females with ACS: Worse Prognosis in 2-years Follow-up
}

\author{
Paola Leocádio, ${ }^{10}$ Penélope Menta, ${ }^{1}$ Melissa Dias, ${ }^{1}$ Júlia Fraga, ${ }^{1}$ Alessandra Goulart, ${ }^{2}$ Itamar Santos, ${ }^{2,3}$ \\ Paulo Lotufo, 2,3 Isabela Bensenor, ${ }^{2,3}$ Jacqueline Alvarez-Leite ${ }^{1}$ \\ Departamento de Bioquímica e Imunologia - Universidade Federal de Minas Gerais, ${ }^{1}$ Belo Horizonte, MG - Brasil \\ Centro de Pesquisa Clínica e Epidemiológica do Hospital Universitário da Universidade de São Paulo, ${ }^{2}$ São Paulo, SP - Brasil \\ Departamento de Clínica Médica da Faculdade de Medicina da Universidade de São Paulo, ${ }^{3}$ São Paulo, SP - Brasil
}

\section{Resumo}

Fundamento: Vários marcadores têm sido avaliados quanto a um potencial impacto nas decisões clínicas ou na predição de mortalidade na síndrome coronariana aguda (SCA), incluindo Netrina-1 e IL-1 $\beta$.

Objetivo: Examinamos o valor prognóstico de Netrina-1 e IL-1 $\beta$ em pacientes com SCA (2 anos de acompanhamento).

Métodos: Avaliamos Netrina-1, IL-1 $\beta$ e outros fatores de risco em amostras de soro de 803 pacientes. Curvas de Kaplan-Meier e regressão de Cox foram usadas para análise de óbito por todas as causas, óbito por doenças cardiovasculares (DCV) e desfecho combinado de infarto agudo do miocárdio (IAM) fatal ou novo IAM não fatal, considerando p < 0,05.

Resultados: Houve 115 óbitos por todas as causas, 78 óbitos por DCV e 67 eventos no desfecho combinado. Níveis de Netrina-1 acima da mediana ( $>44,8 \mathrm{pg} / \mathrm{mL}$ ) foram associados a pior prognóstico (óbito por todas as causas e por DCV) em mulheres idosas, mesmo após o ajuste do modelo (HR: 2,08, $p=0,038$ e HR: 2,68, $p=0,036$ ). Níveis de IL-1 $\beta$ acima da mediana ( $>13,4 \mathrm{pg} / \mathrm{mL}$ ) em mulheres idosas foram associados a risco aumentado para todos os desfechos após o ajuste (todas as causas - HR: 2,03, p = 0,031; DCV - HR: 3,01, p = 0,013; desfecho combinado - HR: 3,05, p = 0,029). Para homens, não foram observadas associações entre Netrina-1 ou IL-1 $\beta$ e os desfechos.

Conclusão: Níveis séricos elevados de Netrina-1 e IL-1 $\beta$ mostraram associação significativa com pior prognóstico em idosas do sexo feminino. Eles podem ser úteis como indicadores prognósticos em SCA. (Arq Bras Cardiol. 2020; 114(3):507-514)

Palavras-chave: Síndrome Coronariana Aguda/fisiopatologia; Nitrina-1; Interleucina- 1 beta; Remodelamento Atrial; Hipertensão; Diabetes Mellitus; Dislipidemias; Acidente Vascular Cerebral; Idoso; Mulheres.

\begin{abstract}
Background: Several markers have been evaluated for a potential impact on clinical decisions or mortality prediction in acute coronary syndrome (ACS), including Netrin-1 and IL-1 $\beta$ that have been associated with cardiovascular disease.

Objective: Our study examined the prognostic value of Netrin-1 and IL-1 $\beta$ in patients with ACS (2-year follow-up).

Methods: We evaluate Netrin-1, IL-1 $\beta$ and other risk factors in the serum sample of 803 patients. Kaplan-Meier curves and Cox regression were used for the analysis of all-cause mortality, cardiovascular mortality, and a combined outcome of fatal myocardial infarction (MI) or new non-fatal MI, considering p-value $<0.05$.

Results: There were 115 deaths from all causes, 78 deaths due to cardiovascular causes and 67 events in combined outcomes. Netrin-1 levels above the median (>44.8 pg/mL) were associated with a worse prognosis (all-cause mortality and cardiovascular mortality) in elderly females, even after model adjustment (HR: 2.08, $p=0.038$ and HR: 2.68, $p=0.036$ ). IL-1 $\beta$ levels above the median (>13.4 pg/mL) in elderly females were associated with increased risk of all outcomes after adjustment (all-cause mortality - HR: 2.03, $p=0.031$; cardiovascular mortality - HR: 3.01, $p=0.013$; fatal MI or new non-fatal MI - HR: 3.05, $p=0.029$ ). For males, no associations were observed between Netrin-1 or IL-1 $\beta$ and outcomes. Conclusion: High serum levels of Netrin-1 and IL-1 $\beta$ showed significant association with worse prognosis in elderly females. They may be useful as prognostic indicators in ACS. (Arq Bras Cardiol. 2020; 114(3):507-514)
\end{abstract}

Keywords: Acute Coronary Syndrome/physiopathology; Netrin-1; Interleukin-1 beta; Atrial Remodeling; Hypertension; Diabetes Mellitus; Dyslipidemias; Stroke; Aged; Women.

Full texts in English - http://www.arquivosonline.com.br

Correspondência: Paola Leocádio •

Departamento de Bioquímica e Imunologia - Universidade Federal de Minas Gerais - Avenida Antonio Carlos, 6627. CEP 31270-901, Pampulha,

Belo Horizonte, MG - Brasil

E-mail: paolaleocadio@yahoo.com.br

Artigo recebido em 22/01/2019, revisado em 23/03/2019, aceito em 03/06/2019

DOI: https://doi.org/10.36660/abc.20190035 


\section{Introdução}

A doença arterial coronariana (DAC) é a principal causa de morte e anos de vida perdidos. ${ }^{1}$ Responsável pelo maior número de óbitos no Brasil, a DAC tem alta prevalência e prognóstico desfavorável. ${ }^{2}$ Apesar da redução na mortalidade por síndrome coronariana aguda (SCA) observada nas últimas décadas, ${ }^{1}$ estima-se que aproximadamente $14 \%$ dos pacientes que tiveram infarto agudo do miocárdio (IAM) morrerão em decorrência do mesmo. ${ }^{3} \mathrm{O}$ risco de doença e morte é 1,5 a 15 vezes maior para pacientes que sobrevivem ao estágio agudo do IAM do que para a população geral. ${ }^{3}$ Daqueles que têm um primeiro IAM, aproximadamente $17 \%$ dos homens e $21 \%$ das mulheres com $\geq 45$ anos terão um IAM recorrente ou DAC fatal dentro de cinco anos. ${ }^{3}$

A inflamação é um fator importante na fisiopatologia da SCA, bem como na remodelação cardíaca após IAM. Diversos marcadores foram avaliados quanto a um impacto potencial nas decisões clínicas ou na predição da mortalidade. ${ }^{4}$ Recentemente, moléculas de orientação neuronal, especialmente Netrina-1, foram identificadas como importantes moduladoras da aterosclerose, embora seu papel específico (protetor ou deletério) ainda seja controverso. ${ }^{5-7}$ A Netrina-1 faz parte de uma família de proteínas estruturalmente semelhantes às lamininas, que são componentes estruturais da membrana basal dos tecidos. ${ }^{8} \mathrm{O}$ papel da Netrina-1 na doença cardiovascular (DCV) e na inflamação é uma área emergente de estudo. ${ }^{5}$

A interleucina-1 (IL-1) é um dos principais mediadores da coagulação induzida pela inflamação. ${ }^{9}$ A IL-1 $\beta$ é capaz de induzir a expressão de outras moléculas que favorecem o recrutamento de células inflamatórias para a lesão e injúria tecidual. ${ }^{10}$ Níveis elevados de IL-1 foram descritos no IAM. ${ }^{11,12}$ Recentemente, um ensaio clínico randomizado entre pessoas que sofreram um infarto do miocárdio mostrou que o canakinumab, um agente que bloqueia a IL-1 $\beta$, reduziu a incidência de doença coronariana não fatal, eventos de acidente vascular cerebral não fatal e morte por DCV. ${ }^{13}$

Apesar da importância dessas duas moléculas na SCA, estudos avaliando o valor prognóstico das mesmas são escassos. Nosso objetivo foi avaliar o papel dessas moléculas como preditores de prognóstico em um acompanhamento de 2 anos.

\section{Métodos}

\section{Desenho experimental}

Os participantes fazem parte do estudo "ERICO" (Estratégia de Registro de Insuficiência Coronariana), descrito detalhadamente em trabalhos anteriores. ${ }^{14,15}$ Resumidamente, trata-se de um estudo de coorte prospectivo que incluiu indivíduos internados para tratamento de SCA no Hospital Universitário da Universidade de São Paulo (HU-USP), hospital de ensino com 260 leitos localizado no Butantã, São Paulo, Brasil, de fevereiro de 2009 a dezembro de 2013. O protocolo do estudo está de acordo com a Declaração de Helsinque. Este estudo foi aprovado pelo Comitê de Ética em Pesquisa (CEP-HU/USP 866/08) e todos os pacientes assinaram o Termo de Consentimento Livre e Esclarecido.

O IAM foi definido pela presença de sintomas compatíveis com isquemia miocárdica dentro de 24 horas da admissão hospitalar e nível de troponina I acima do valor do percentil
99 com um coeficiente de variação $<10 \%$. O infarto agudo do miocárdio com supradesnivelamento do segmento ST (IAMCSST) foi definido pelos critérios de IAM, além de: (a) a presença de supradesnivelamento do segmento ST persistente $\geq 1 \mathrm{~mm}$ em dois eletrocardiogramas contíguos (ECG de eletrodo) ou (b) novo (ou supostamente novo) bloqueio de ramo esquerdo (BRE). O infarto agudo do miocárdio sem supradesnivelamento do segmento ST (IAMSSST) foi definido pelos critérios para IAM mais ausência de supradesnivelamento do segmento ST persistente $\geq 1 \mathrm{~mm}$ em dois ECGs contíguos e de novo ou supostamente novo BRE. A angina instável (AI) foi definida como a presença de sintomas compatíveis com isquemia miocárdica nas últimas 24 horas, ausência de diagnóstico de IAM e pelo menos um dos cinco critérios a seguir: (a) história prévia de DAC; (b) estratificação positiva de doença isquêmica invasiva ou não invasiva do coração; (c) alterações dinâmicas ou evolutivas do ECG; (D) troponina I > 0,4 ng/mL (assegurando níveis de troponina I acima do percentil 99, independentemente do kit utilizado) ou (e) concordância no diagnóstico de Al entre dois médicos independentes.

\section{Coleta de dados e desfechos}

Após seis meses e anualmente por dois anos após a admissão hospitalar, todos os indivíduos foram contatados por telefone para atualizar as informações do estado vital, incluindo desfechos cardiovasculares fatais e não fatais. Sempre que um participante relatou um possível novo evento de IAM, novos procedimentos de investigação foram iniciados para confirmar o evento.

Os desfechos avaliados foram: óbito por todas as causas, óbito por DCV e o desfecho combinado (IAM fatal ou novo IAM não fatal). A estratégia para coletar e classificar os dados de mortalidade, incluindo a busca por registros oficiais de óbitos, foi detalhada em estudo anterior. ${ }^{15}$ Nos casos em que não foi possível determinar a causa da morte, os dados foram censurados para todos os desfechos, com exceção da morte por todas as causas.

Durante a fase hospitalar, entrevistadores treinados coletaram dados relacionados às características sociodemográficas, fatores de risco cardiovascular e medicação, conforme descrito anteriormente..$^{15}$ Amostras de sangue foram coletadas dentro de 24 horas da admissão. Análises de glicose plasmática, triglicérides, colesterol total e HDL foram realizadas no HU-USP. O colesterol LDL foi calculado pela equação de Friedewald. ${ }^{16}$ As concentrações de Netrina-1 e IL-1 $\beta$ na admissão foram avaliadas por Enzyme-Linked Immunosorbent Assay (ELISA), seguindo as instruções do kit (Netrina-1: SEB827HU; USCN Life Science Inc., Wuhan, China e IL-1 $\beta$ : 88- 7010-88 eBioscience Inc., San Diego, CA, EUA). Os pacientes foram classificados de acordo com as concentrações de Netrina-1 e IL-1 $\beta$ nos grupos "baixa" e "alta", se sua concentração estivesse abaixo ou acima da mediana.

\section{Análise estatística}

Os dados foram avaliados quanto à normalidade usando o teste de Kolmogorov-Smirnov. Ostestes qui-quadradoe Mann-Whitney (todas as variáveis contínuas apresentaram distribuição não-paramétrica) foram utilizados para comparar os grupos. Os valores foram expressos como mediana (intervalo interquartil) ou n (\%). Curvas de Kaplan-Meier foram utilizadas, e o teste 
log-rank foi utilizado para avaliar a diferença entre os grupos. As estimativas de risco (razões de risco com seus respectivos intervalos de confiança de 95\%) para os eventos foram calculadas usando regressão de Cox. Além de Netrina-1 e IL-1 $\beta$, as seguintes variáveis foram usadas para construir modelos: idade, tipo de SCA, diabetes, hipertensão e dislipidemia. Um valor de $\mathrm{p}<0,05$ foi considerado significativo.

Os programas SPSS (IBM SPSS Statistics para Windows, versão 22.0, Armonk, NY: IBM Corp.) e GraphPad Prism (versão 5.01 para Windows, San Diego, Califórnia: GraphPad Software) foram usados para realizar as análises.

\section{Resultados}

Um total de 803 pacientes foram incluídos neste estudo, incluindo 333 mulheres e 470 homens. Comparando as principais características dos grupos de homens e mulheres, observamos que as mulheres eram mais velhas e apresentavam maior concentração de HDL que os homens. As mulheres também foram mais afetadas pela hipertensão, diabetes e dislipidemia (Tabela 1). O tipo mais frequente de SCA entre os grupos foi o IAMSSST (cerca de $40 \%$ dos casos), seguido por AI e IAMCSST, que tiveram frequência semelhante (cerca de $30 \%$ cada).
Durante os dois anos de acompanhamento, houve 115 mortes por todas as causas (65 homens e 50 mulheres), incluindo 78 mortes $(67,8 \%)$ por causas cardiovasculares. Também identificamos 67 casos de IAM (fatal ou não fatal) neste mesmo seguimento. Como a idade é um fator importante envolvido na taxa de mortalidade, analisamos separadamente em mulheres e homens jovens e com mais de 60 anos.

Para avaliar um possível papel de Netrina- 1 e IL-1 $\beta$ como marcadores prognósticos, comparamos a frequência de SCA nos pacientes com níveis de Netrina-1 e IL-1 $\beta$ acima e abaixo da respectiva mediana. Não houve associações entre os níveis de Netrina-1 e IL-1 $\beta$ e todos os desfechos para homens independentemente de sua idade (dados não mostrados). Por esse motivo, focamos nossa investigação no grupo de mulheres (333 pacientes). As principais características do grupo de mulheres (jovens e idosas) são mostradas na Tabela 2.

Na admissão, as mulheres apresentaram valores semelhantes de índice de massa corporal, concentrações séricas de glicose, triglicerídeos e colesterol $\mathrm{HDL}$, independentemente do grupo etário. A frequência de fatores de risco como hipertensão, dislipidemia e diabetes foi maior em mulheres idosas. No entanto, os níveis de colesterol LDL foram menores nas idosas. Fumantes atuais foram mais frequentes em mulheres jovens, enquanto mais de $50 \%$ das mulheres idosas nunca

Tabela 1 - Características gerais na fase hospitalar em homens e mulheres

\begin{tabular}{|c|c|c|c|}
\hline Parâmetro & Homens $(n=470)$ & Mulheres $(n=333)$ & $\mathrm{p}$ \\
\hline Idade & $60(52-71)$ & $65(56-76)$ & $<0,0001$ \\
\hline IMC & $26,8(23,8-29,6)$ & $26,8(24,0-30,9)$ & 0,128 \\
\hline Tipo SCA & & & 0,027 \\
\hline IAMSSST & $191(40,6)$ & $142(42,6)$ & \\
\hline IAMCSST & $147(31,3)$ & $77(23,1)$ & \\
\hline $\mathrm{Al}$ & $132(28,1)$ & $114(34,2)$ & \\
\hline \multicolumn{4}{|l|}{ Tabagismo } \\
\hline Atual & $141(31,3)$ & $85(27,2)$ & $<0,0001$ \\
\hline Passado & $198(43,9)$ & $82(26,3)$ & \\
\hline Nunca & $112(24,8)$ & $145(46,5)$ & \\
\hline Hipertensão & $339(73,7)$ & $267(80,9)$ & 0,018 \\
\hline Diabetes & $156(34,7)$ & $148(45,0)$ & 0,004 \\
\hline Dislipidemia & $197(48,0)$ & $181(60,7)$ & 0,001 \\
\hline Glicose & $125,0(101,0-157,0)$ & $124,0(103,0-175,0)$ & 0,652 \\
\hline Triglicerídeos & $132,0(94,0-190,3)$ & $126,0(97,0-183,0)$ & 0,685 \\
\hline Colesterol total & $171,5(141,0-205,0)$ & $170,0(139,0-204,0)$ & 0,720 \\
\hline Colesterol HDL & $35,0(30,0-44,0)$ & $39,0(32,0-46,5)$ & $<0,0001$ \\
\hline Colesterol LDL & $102,5(77,0-134,3)$ & $99,0(77,0-124,3)$ & 0,386 \\
\hline Netrina-1 & $44,8(34,2-65,8)$ & $44,8(34,8-62,8)$ & 0,813 \\
\hline IL-1 $1 \beta$ & $15,1(7,4-28,8)$ & $13,8(7,1-29,7)$ & 0,536 \\
\hline
\end{tabular}

Mediana (intervalo interquartil) ou (\%). Al: angina instável. HDL: lipoproteína de alta densidade. IAMCSST: infarto do miocárdio com supradesnivealmento do segmento ST. IAMSSST: infarto do miocárdio sem supradesnivealmento do segmento ST. IL-1ß: interleucina - 1 beta. IMC: índice de massa corporal em kg/m². LDL: lipoproteína de baixa densidade. SCA: síndrome coronariana aguda. Dados de glicose plasmática, triglicerídeos, colesterol total, HDL e LDL são apresentados em mg/dL. Netrina-1 e IL-1 $\beta$ são apresentados em $\mathrm{pg} / \mathrm{mL}$. Teste de Mann-Whitney ou teste de qui-quadrado. $p$-valor comparando homens e mulheres. 
Tabela 2 - Características gerais na fase hospitalar em mulheres jovens ( $<60$ anos) e idosas ( $>60$ anos)

\begin{tabular}{|c|c|c|c|c|}
\hline Parâmetro & Total $(n=333)$ & Jovens $(<60$ anos) $(n=111)$ & Idosas $(>60$ anos) $(n=222)$ & $\mathrm{p}$ \\
\hline IMC & $26,8(24,0-30,9)$ & $26,8(24,5-31,1)$ & $26,7(23,8-30,8)$ & 0,532 \\
\hline Tipo SCA & & & & 0,031 \\
\hline IAMSSST & $142(42,6)$ & $40(36,0)$ & $102(45,9)$ & \\
\hline IAMCSST & $77(23,1)$ & $35(31,5)$ & $42(18,9)$ & \\
\hline $\mathrm{Al}$ & $114(34,2)$ & $36(32,4)$ & $78(35,1)$ & \\
\hline Tabagismo & & & & $<0,0001$ \\
\hline Atual & $85(27,2)$ & $47(44,3)$ & $38(18,4)$ & \\
\hline Passado & $82(26,3)$ & $28(26,4)$ & $54(26,2)$ & \\
\hline Nunca & $145(46,5)$ & $31(29,2)$ & $114(55,3)$ & \\
\hline Hipertensão & $267(80,9)$ & $79(72,5)$ & $188(85,1)$ & 0,006 \\
\hline Diabetes & $148(45,0)$ & $39(35,8)$ & $109(49,5)$ & 0,018 \\
\hline Dislipidemia & $181(60,7)$ & $48(48,5)$ & $133(66,8)$ & 0,002 \\
\hline Glicose & $124,0(103,0-175,0)$ & $121,0(103,0-163,0)$ & $128,0(104,0-180,0)$ & 0,381 \\
\hline Triglicerídeos & $126,0(97,0-183,0)$ & $151,0(97,0-201,0)$ & $122,0(93,5-174,0)$ & 0,080 \\
\hline Colesterol total & $170,0(139,0-204,0)$ & $185,0(157,5-215,3)$ & $161,0(134,0-196,0)$ & 0,002 \\
\hline Colesterol HDL & $39,0(32,0-46,5)$ & $36,0(32,0-45,0)$ & $41,0(33,0-47,2)$ & 0,065 \\
\hline Colesterol LDL & $99,0(77,0-124,3)$ & $114,0(89,0-134,0)$ & $93,0(72,0-118,0)$ & 0,0004 \\
\hline Netrina-1 & $44,8(34,8-62,8)$ & $44,8(33,8-65,8)$ & $44,8(34,7-65,0)$ & 0,861 \\
\hline IL-1 $\beta$ & $13,8(7,1-29,7)$ & $15,5(7,9-49,7)$ & $13,4(7,1-24,1)$ & 0,037 \\
\hline
\end{tabular}

Mediana (intervalo interquartil) ou $n$ (\%). Al: angina instável. HDL: lipoproteína de alta densidade. IAMCSST: infarto do miocárdio com supradesnivealmento do segmento ST. IAMSSST: infarto do miocárdio sem supradesnivealmento do segmento ST. IL-1ß: interleucina - 1beta. IMC: índice de massa corporal em $\mathrm{kg} / \mathrm{m}^{2}$. LDL: lipoproteína de baixa densidade. SCA: síndrome coronariana aguda. Dados de glicose plasmática, triglicerídeos, colesterol total, HDL e LDL são apresentados em mg/dL. Netrina-1 e IL-1 $\beta$ são apresentados em pg/mL. Teste de Mann-Whitney ou teste de qui-quadrado.

fumou (Tabela 2). Não encontramos diferenças na mediana de Netrina-1 entre os grupos etários. No entanto, a mediana de IL-1 $\beta$ foi maior no grupo mais jovem.

As associações entre Netrina-1 ou IL1- $\beta$ baixa e alta e os desfechos, de acordo com a faixa etária, foram apresentadas na Tabela 3 e na Tabela 4, respectivamente. O número de mortes por todas as causas foi muito baixo ( 3 mortes) nas mulheres com menos de 60 anos e apenas 6 casos do desfecho combinado, dificultando análises confiáveis neste grupo. No entanto, no grupo mais velho (> 60 anos), encontramos associações entre níveis elevados de Netrina-1 e mortes por todas as causas e causas cardiovasculares. Uma associação entre IL-1 $\beta$ alta e morte por DCV também foi encontrada entre as mulheres idosas $(p=0,034)$.

Os dados mostrando um pior prognóstico em mulheres idosas com níveis elevados de Netrina-1 e IL-1 $\beta$ foram confirmados pelas curvas de Kaplan-Meier. Níveis elevados de Netrina-1 resultaram em uma menor taxa de sobrevivência quando se considera a mortalidade por todas as causas $(p=0,011$, Figura 1A) e também considerando apenas mortes por DCV ( $p=0,024$, Figura 1B). Houve tendência de associação a IAM fatal ou novo IAM não fatal $(p=0,067$, Figura 1C). Níveis elevados de IL-1 $\beta$ também mostraram uma menor taxa de sobrevivência quando consideradas mortes por DCV ( $p=0,031$, Figura 1E), com tendência a associação com o desfecho combinado ( $p=0,064$, Figura 1F).
A análise das razões de risco (Tabela 5) mostrou um aumento no risco de morte por todas as causas para o grupo Netrina-1 alta, que permaneceu significativo no modelo ajustado. Os mesmos resultados foram observados para o risco de morte por DCV.

Considerando os níveis elevados de IL-1 $\beta$, não encontramos HR significativa no modelo cru para mortalidade por todas as causas e para o desfecho combinado (Tabela 5). No entanto, foi observada HR significativa para mortalidade por todas as causas e desfecho combinado no modelo ajustado. Observamos também um risco aumentado de morte por causas cardiovasculares para o grupo IL-1 $\beta$ alta, mesmo após o ajuste do modelo.

\section{Discussão}

Este trabalho é pioneiro na avaliação do valor prognóstico da Netrina-1 na SCA e apresenta novas informações sobre o valor prognóstico da IL-1 $\beta$ nessa condição.

Em nosso estudo, observamos uma associação entre níveis elevados de Netrina-1 e pior prognóstico quando foram analisados óbito por todas as causas e por DCV em mulheres idosas.

A Netrina-1 plasmática é um biomarcador diagnóstico de muitos tipos de câncer. ${ }^{17-19}$ Verificou-se que a maior expressão gênica ou concentração de Netrina-1 nesses tecidos é associada a um pior prognóstico, provavelmente relacionado aos efeitos 
Tabela 3 - Desfechos de acordo com os níveis de Netrina-1 em mulheres

\begin{tabular}{lccccccccc}
\hline & \multicolumn{3}{c}{ Mulheres - Total } & & \multicolumn{2}{c}{$<6$ anos } & & \multicolumn{2}{c}{$>60$ anos } \\
\hline Acompanhamento de 2 anos & $\begin{array}{c}\text { Netrina-1 } \\
\text { baixa }\end{array}$ & $\begin{array}{c}\text { Netrina-1 } \\
\text { alta }\end{array}$ & $\mathbf{p}$ & $\begin{array}{c}\text { Netrina-1 } \\
\text { baixa }\end{array}$ & $\begin{array}{c}\text { Netrina-1 } \\
\text { alta }\end{array}$ & $\mathbf{p}$ & $\begin{array}{c}\text { Netrina-1 } \\
\text { baixa }\end{array}$ & $\begin{array}{c}\text { Netrina-1 } \\
\text { alta }\end{array}$ & $\begin{array}{c}\mathbf{p} \\
\text { Óbito por todas as causas }\end{array}$ \\
\hline Óbito por DCV & $18(36,0)$ & $32(64,0)$ & 0,021 & $2(66,7)$ & $1(33,3)$ & 0,612 & $16(34,0)$ & $31(66,0)$ & 0,011 \\
IAM fatal ou novo IAM não fatal & $12(35,3)$ & $22(64,7)$ & 0,052 & $2(66,7)$ & $1(33,3)$ & 0,612 & $10(32,3)$ & $21(67,7)$ & 0,029 \\
\hline
\end{tabular}

$n(\%)$. DCV: doenças cardiovasculares. IAM: infarto agudo do miocárdio. Teste do qui-quadrado.

Tabela 4 - Desfechos de acordo com os níveis de IL-1 $\beta$ em mulheres

\begin{tabular}{lccccccccc}
\hline & \multicolumn{3}{c}{ Mulheres - Total } & & $<6$ anos & & \multicolumn{3}{c}{$>60$ anos } \\
\hline Acompanhamento de 2 anos & IL-1 $\beta$ baixa & IL-1 $\beta$ alta & $\mathbf{p}$ & IL-1 $\beta$ baixa & IL-1 $\beta$ alta & p & IL-1 $\beta$ baixa & IL-1 $\beta$ alta & p \\
\hline Óbito por todas as causas & $21(42,0)$ & $29(58,0)$ & 0,221 & $0(0,0)$ & $3(100,0)$ & 0,118 & $19(40,4)$ & $28(59,6)$ & 0,140 \\
Óbito por DCV & $12(35,3)$ & $22(64,7)$ & 0,071 & $0(0,0)$ & $3(100,0)$ & 0,118 & $10(32,3)$ & $21(67,7)$ & 0,034 \\
IAM fatal ou novo IAM não fatal & $10(35,7)$ & $18(64,3)$ & 0,117 & $2(33,3)$ & $4(66,7)$ & 0,438 & $7(31,8)$ & $15(68,2)$ & 0,075 \\
\hline
\end{tabular}

$n$ (\%). DCV: doenças cardiovasculares. IAM: infarto agudo do miocárdio. IL-1ß: Interleucina - 1 beta. Teste do qui-quadrado.

antiapoptóticos e angiogênicos da molécula. No entanto, o papel da Netrina-1 na aterosclerose e na remodelação cardíaca após o IAM ainda é controverso. Foi descrito que Netrina-1 poderia promover ou proteger contra a aterosclerose, dependendo das condições ambientais. ${ }^{20}$ Níveis endógenos reduzidos de Netrina-1 também podem levar a efeitos deletérios, uma vez que fatores pró-aterogênicos podem reduzir a expressão dessa molécula. ${ }^{20}$ Em modelos de IAM, a administração de netrina-1 reduziu a gravidade da lesão miocárdica quando comparada aos controles não suplementados..$^{21,22}$

Em nosso estudo, é possível que o grupo "Netrina-1 alta" seja composto por pacientes que tiveram um evento mais grave de SCA. Nossa hipótese é que o nível de Netrina-1 aumenta em casos mais graves de SCA. Esta hipótese baseia-se em estudos que indicam que a expressão de Netrina-1 é induzida após lesão celular e pode ser usada como um biomarcador para danos ou doenças de órgãos, ${ }^{23}$ como visto na cirurgia cardíaca. ${ }^{24}$ Além disso, a hipóxia, uma condição intimamente ligada à aterosclerose e à SCA, também é um indutor da expressão da Netrina-1. ${ }^{25}$ Ainda, Van Gils et al. ${ }^{6}$ observaram aumento da expressão da molécula em macrófagos carregados de colesterol promovendo a retenção dessas células in vitro, o que poderia contribuir para uma evolução mais rápida da placa aterosclerótica e, consequentemente, aumentar a chance de formação de trombo e ocorrência de infarto.

Esses fatores nos levam a acreditar que o maior número de desfechos desfavoráveis, bem como o maior risco de morte por todas as causas e por DCV observados em mulheres idosas com níveis mais elevados de Netrina- 1 estão associados à gravidade do evento e ao maior grau de inflamação (como sugerido pelos altos níveis de IL-1 $\beta$ ) que podem ter contribuído para um pior prognóstico.

Em relação a IL-1 $\beta$, níveis elevados desta e de outras citocinas pró-inflamatórias já foram identificados em pacientes com SCA. ${ }^{26}$ No entanto, poucos estudos foram direcionados ao valor prognóstico dessa citocina. ${ }^{27,28}$ Corroborando nossos resultados, esses estudos sugerem que níveis mais altos de IL-1 $\beta$ foram observados em pacientes com SCA que sofreram novos eventos durante o acompanhamento.

A IL-1 $\beta$ é capaz de aumentar a expressão de moléculas que contribuem para a ruptura da placa e formação de trombos, culminando na ocorrência de SCA. ${ }^{10,29}$ Assim, níveis mais elevados de IL-1 $\beta$ sérica poderiam refletir inflamação exacerbada, favorecendo a ocorrência de complicações cardiovasculares.

Níveis mais altos de IL-1 $\beta$ sugerem uma inflamação exacerbada que pode prejudicar o remodelamento cardíaco. A remodelação adversa após o IAM é a base estrutural da insuficiência cardíaca isquêmica. Embora quantidades adequadas de IL-1 $\beta$ e outras citocinas inflamatórias sejam essenciais na fase inicial do remodelamento, a diminuição dos níveis de citocinas é necessária para promover uma cicatrização efetiva. ${ }^{30}$ Foi descrito que níveis elevados de IL-1 $\beta$ até dois meses após o infarto em pacientes com IAMCSST estavam fortemente associados à piora da função cardíaca após um ano de acompanhamento. ${ }^{31}$ Além disso, a citocina foi um forte preditor de hipertrofia ventricular esquerda, importante na predição da morbidade e mortalidade cardiovascular. ${ }^{31}$

Vários fatores podem ajudar a entender a ausência de associação com pior prognóstico encontrada em homens e mulheres mais jovens. Os tecidos cardíacos e vasculares são influenciados por hormônios como estrógeno e testosterona, variando de acordo com sexo e idade. ${ }^{32,33}$ Mulheres mais velhas têm maior massa do ventrículo esquerdo do que homens, devido a fatores que indicam menor capacidade arterial, como redução da espessura da parede da carotída. ${ }^{33}$

A relação entre citocinas inflamatórias e gênero ainda não foi elucidada, embora diferenças na concentração sejam observadas na literatura. ${ }^{32}$ Estudos indicam que a concentração de IL- $\beta$ é maior no sexo masculino. ${ }^{33,34}$ Além disso, níveis de citocinas estão inversamente relacionados 
A
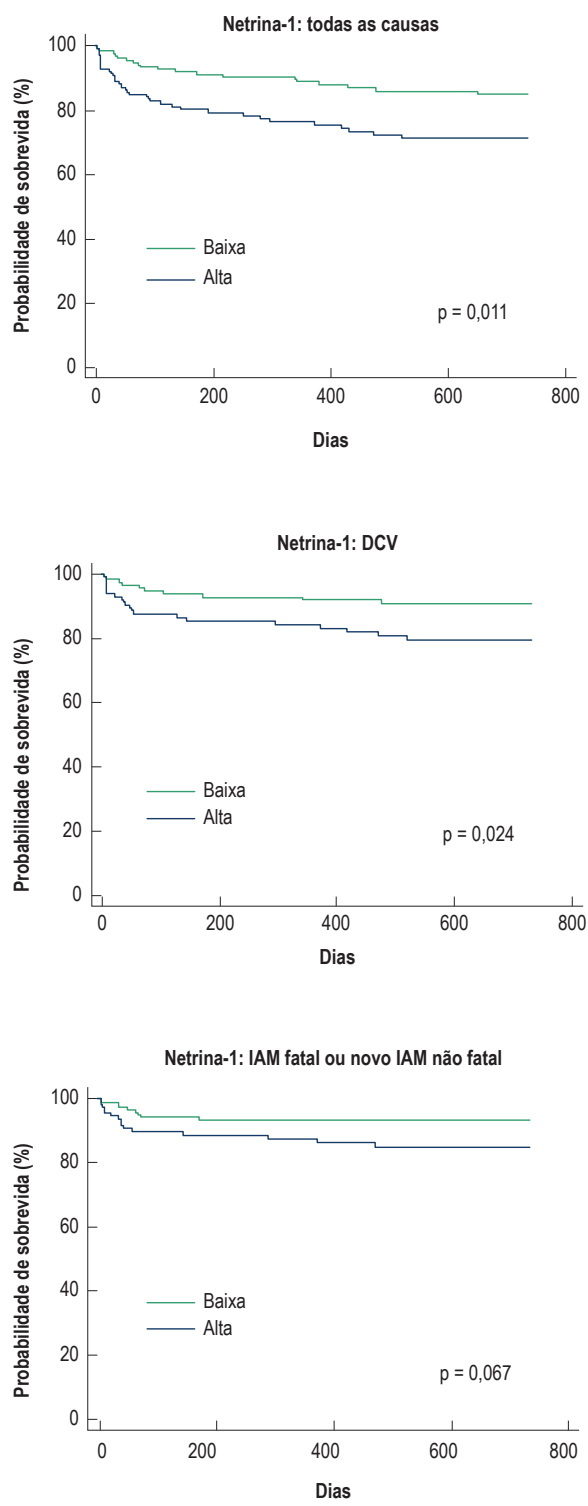
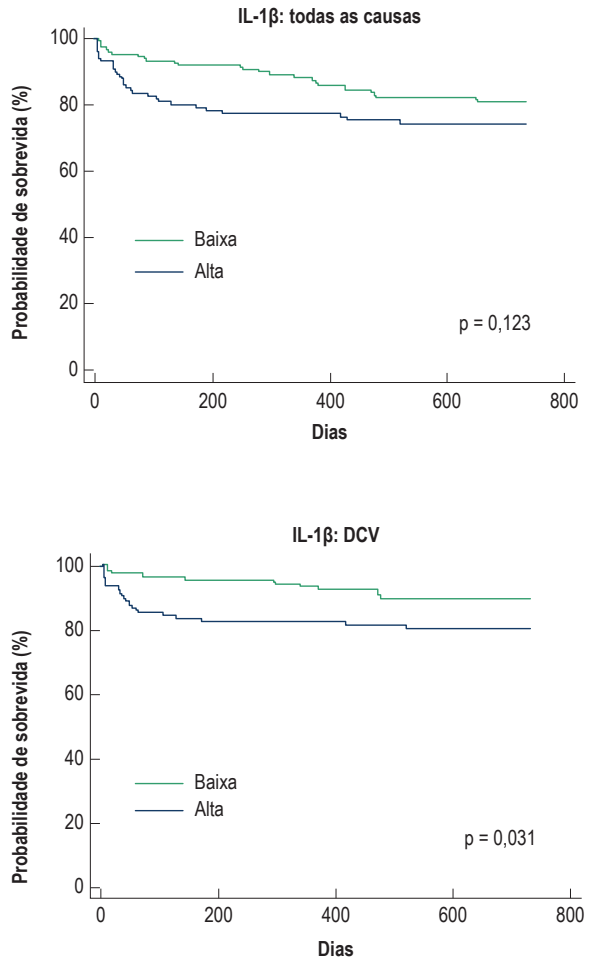

$\mathrm{F}$

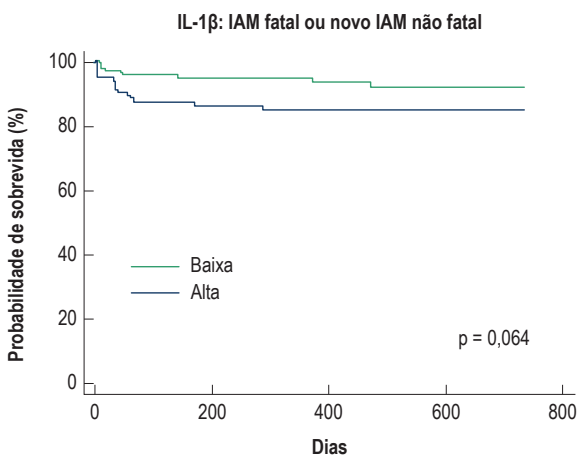

Figura 1 - Curvas de Kaplan-Meier para Netrina-1 $(A-C)$ e IL-1B (D-F) em mulheres idosas (> 60 anos) no acompanhamento de dois anos. IL-1B: Interleucina - 1beta; DCV: doença cardiovascular; IAM: infarto agudo do miocárdio.

Tabela 5 - Razão de risco (HR) para os níveis de Netrina-1 e IL-1 $\beta$ e óbito por todas as causas, óbito por doenças cardiovasculares e desfecho combinado de IAM fatal ou novo IAM não fatal em mulheres idosas (> 60 anos)

\begin{tabular}{|c|c|c|c|c|c|c|}
\hline & \multicolumn{2}{|c|}{ Óbito por todas as causas } & \multicolumn{2}{|c|}{ Óbito por DCV } & \multicolumn{2}{|c|}{ IAM fatal ou novo IAM não fatal } \\
\hline & HR (IC 95\%) & $\mathrm{p}$ & HR (IC 95\%) & $\mathrm{p}$ & HR (IC 95\%) & $\mathrm{p}$ \\
\hline & \multicolumn{6}{|c|}{ Netrina-1 } \\
\hline Crua & $2,15(1,17-3,93)$ & 0,013 & $2,31(1,09-4,92)$ & 0,029 & $2,25(0,92-5,54)$ & 0,075 \\
\hline \multirow[t]{2}{*}{ Ajustada * } & $2,08(1,04-4,16)$ & 0,038 & $2,68(1,06-6,74)$ & 0,036 & $1,82(0,65-5,07)$ & 0,247 \\
\hline & \multicolumn{6}{|c|}{ IL-1 $\beta$} \\
\hline Crua & $1,57(0,87-2,81)$ & 0,127 & $2,23(1,05-4,75)$ & 0,036 & $2,27(0,92-5,58)$ & 0,073 \\
\hline Ajustada * & $2,03(1,06-3,89)$ & 0,031 & $3,01(1,26-7,17)$ & 0,013 & $3,05(1,12-8,32)$ & 0,029 \\
\hline
\end{tabular}

HR: razão de risco. IAM: infarto agudo do miocárdio. IC: intervalo de confiança. IL-1ß: Interleucina - 1 beta. *Ajustado por idade, tipo de síndrome coronariana aguda, diabetes, hipertensão e dislipidemia. 
à idade, como visto em nosso trabalho e na literatura. ${ }^{35}$ A literatura não fornece dados sobre diferenças de sexo para Netrina-1. Como não observamos diferenças estatísticas entre os sexos nos níveis dos marcadores, nossa hipótese é de que no sexo feminino os níveis destes eram mais altos que os normais. Esse possível aumento pode estar relacionado à maior frequência de fatores de risco cardiovasculares neste grupo no presente estudo, fatores que podem levar a um aumento dos níveis de marcadores inflamatórios. ${ }^{36}$ No entanto, mesmo após o ajuste desses fatores, níveis elevados de Netrina 1 e IL-1 $\beta$ permaneceram associados a pior prognóstico, demonstrando que esses marcadores inflamatórios estão independentemente associados a pior prognóstico e podem estar relacionados à redução da capacidade arterial já observada em mulheres idosas, quando comparados aos homens.

Apontamos como limitações do estudo sua característica unicêntrica e a ausência de um grupo controle. Ainda, não coletamos dados do período pré-evento, o que nos permitiria determinar a variação na concentração do marcador após a SCA.

\section{Conclusão}

Níveis elevados de Netrina-1 e IL-1 $\beta$ estão associados a pior prognóstico em mulheres idosas com SCA. O mecanismo para tal associação pode estar relacionado à manutenção da inflamação e remodelação cardíaca adversa, propiciando novos eventos cardiovasculares.

\section{Contribuição dos autores}

Concepção e desenho da pesquisa e Análise e interpretação dos dados: Leocádio P, Goulart A, Santos I,
Lotufo P, Bensenor I, Alvarez-Leite, J; Obtenção de dados: Leocádio P, Menta P, Dias M, Fraga J, Goulart A, Santos I, Lotufo P, Bensenor I; Análise estatística: Leocádio P, Goulart A, Santos I; Obtenção de financiamento: Lotufo P, Bensenor I; Redação do manuscrito: Leocádio P, Menta P, Dias M, Fraga J; Revisão crítica do manuscrito quanto ao conteúdo intelectual importante: Goulart A, Santos I, Lotufo P, Bensenor I, Alvarez-Leite, J.

\section{Potencial conflito de interesses}

Declaro não haver conflito de interesses pertinentes.

\section{Fontes de financiamento}

O presente estudo foi financiado pela Fundação de Amparo à Pesquisa do Estado de São Paulo e Coordenação de Aperfeiçoamento de Pessoal de Nível Superior.

\section{Vinculação acadêmica}

Este artigo é parte de tese de Doutorado de Paola Leocádio pela Universidade Federal do Minas Gerais.

\section{Aprovação ética e consentimento informado}

Este estudo foi aprovado pelo Comitê de Ética do Hospital Universitário - USP sob o número de protocolo CEP-HU/USP 866/08. Todos os procedimentos envolvidos nesse estudo estão de acordo com a Declaração de Helsinki de 1975, atualizada em 2013. O consentimento informado foi obtido de todos os participantes incluídos no estudo.

\section{Referências}

1. Collaborators GDaH. Global, regional, and national disability-adjusted life-years (DALYs) for 315 diseases and injuries and healthy life expectancy (HALE), 1990-2015: a systematic analysis for the Global Burden of Disease Study 2015. Lancet. 2016;388(10053):1603-58.

2. Ribeiro AL, Duncan BB, BrantLC, Lotufo PA, Mill JG, Barreto SM. Cardiovascular Health in Brazil: Trends and Perspectives. Circulation. 2016;133(4):422-33.

3. Benjamin EJ, Blaha MJ, Chiuve SE, Cushman M, Das SR, Deo R, et al. Heart Disease and Stroke Statistics-2017 Update: A Report From the American Heart Association. Circulation. 2017;135(10):e146-e603.

4. Basra SS, Virani SS, Paniagua D, Kar B, Jneid H. Acute Coronary Syndromes: Unstable Angina and Non-ST Elevation Myocardial Infarction. Heart Fail Clin. 2016;12(1):31-48.

5. Layne K, Ferro A, Passacquale G. Netrin-1 as a novel therapeutic target in cardiovascular disease: to activate or inhibit? Cardiovasc Res. 2015;107(4):410-9.

6. van Gils JM, Derby MC, Fernandes LR, Ramkhelawon B, Ray TD, Rayner KJ, et al. The neuroimmune guidance cue netrin-1 promotes atherosclerosis by inhibiting the emigration of macrophages from plaques. Nat Immunol. 2012;13(2):136-43.

7. Bouhidel JO, Wang P, Siu KL, Li H, Youn JY, Cai H. Netrin-1 improves postinjury cardiac function in vivo via DCC/NO-dependent preservation of mitochondrial integrity, while attenuating autophagy. Biochim Biophys Acta. 2015;1852(2):277-89.

8. Cirulli V, Yebra M. Netrins: beyond the brain. Nat Rev Mol Cell Biol. 2007;8(4):296-306.

9. Libby P, Tabas I, Fredman G, Fisher EA. Inflammation and its resolution as determinants of acute coronary syndromes. Circ Res. 2014;114(12):1867-79.

10. Frangogiannis NG. The immune system and cardiac repair. Pharmacol Res. 2008;58(2):88-111.

11. Dewald O, Ren G, Duerr GD, Zoerlein M, Klemm C, Gersch C, et al. Of mice and dogs: species-specific differences in the inflammatory response following myocardial infarction. Am J Pathol. 2004;164(2):665-77.

12. Ertl G, Frantz S. Healing after myocardial infarction. Cardiovasc Res. 2005;66(1):22-32

13. Ridker PM, Everett BM, Thuren T, MacFadyen JG, Chang WH, Ballantyne C, et al. Antiinflammatory Therapy with Canakinumab for Atherosclerotic Disease. N Engl J Med. 2017;377(12):1119-31.

14. Santos IS, Goulart AC, Brandão RM, Santos RC, Bittencourt MS, Sitnik D, et al. One-year Mortality after an Acute Coronary Event and its Clinical Predictors: The ERICO Study. Arq Bras Cardiol. 2015;105(1):53-64.

15. Goulart AC, Santos IS, Sitnik D, Staniak HL, Fedeli LM, Pastore CA, et al. Design and baseline characteristics of a coronary heart disease prospective cohort: two-year experience from the strategy of registry of acute coronary syndrome study (ERICO study). Clinics (Sao Paulo). 2013;68(3):431-4. 
16. Friedewald WT, Levy RI, Fredrickson DS. Estimation of the concentration of low-density lipoprotein cholesterol in plasma, without use of the preparative ultracentrifuge. Clin Chem. 1972;18(6):499-502.

17. Ramesh G, Berg A, Jayakumar C. Plasma netrin-1 is a diagnostic biomarker of human cancers. Biomarkers. 2011;16(2):172-80.

18. Link BC, Reichelt U, Schreiber M, Kaifi JT, Wachowiak R, Bogoevski D, et al. Prognostic implications of netrin-1 expression and its receptors in patients with adenocarcinoma of the pancreas. Ann Surg Oncol. 2007;14(9):2591-9.

19. Harter PN, Zinke J, Scholz A, Tichy J, Zachskorn C, Kvasnicka HM, et al. Netrin-1 expression is an independent prognostic factor for poor patient survival in brain metastases. PLoS One. 2014:9(3):e92311.

20. van Gils JM, Ramkhelawon B, Fernandes L, Stewart MC, Guo L, Seibert T, et al. Endothelial expression of guidance cues in vessel wall homeostasis dysregulation under proatherosclerotic conditions. Arterioscler Thromb Vasc Biol. 2013;33(5):911-9.

21. ZhangJ, Cai H. Netrin-1 prevents ischemia/reperfusion-induced myocardial infarction via a DCC/ERK1/2/eNOS s1177/NO/DCC feed-forward mechanism. J Mol Cell Cardiol. 2010;48(6):1060-70

22. Mao X, Xing H, Mao A, Jiang H, Cheng L, Liu Y, et al. Netrin-1 attenuates cardiac ischemia reperfusion injury and generates alternatively activated macrophages. Inflammation. 2014;37(2):573-80.

23. Reeves WB, Kwon O, Ramesh G. Netrin-1 and kidney injury. II. Netrin-1 is an early biomarker of acute kidney injury. Am J Physiol Renal Physiol. 2008;294(4):F731-8.

24. Ramesh G, Krawczeski CD, Woo JG, Wang Y, Devarajan P. Urinary netrin-1 is an early predictive biomarker of acute kidney injury after cardiac surgery. Clin J Am Soc Nephrol. 2010;5(3):395-401.

25. Ramkhelawon B, Yang Y, van Gils JM, Hewing B, Rayner KJ, Parathath S, et al. Hypoxia induces netrin-1 and Unc $5 \mathrm{~b}$ in atherosclerotic plaques: mechanism for macrophage retention and survival. Arterioscler Thromb Vasc Biol. 2013;33(6):1180-8.

26. Ozeren A, Aydin M, Tokac M, Demircan N, Unalacak M, Gurel A, et al. Levels of serum IL-1 beta, IL-2, IL-8 and tumor necrosis factor-alpha in patients with unstable angina pectoris. Mediators Inflamm. 2003;12(6):361-5.
27. Kilic T, Ural D, Ural E, Yumuk Z, Agacdiken A, Sahin T, et al. Relation between proinflammatory to anti-inflammatory cytokine ratios and longterm prognosis in patients with non-ST elevation acute coronary syndrome. Heart. 2006;92(8):1041-6.

28. Correia LC, Andrade BB, Borges VM, Clarêncio J, Bittencourt AP, Freitas $R$, et al. Prognostic value of cytokines and chemokines in addition to the GRACE Score in non-ST-elevation acute coronary syndromes. Clin Chim Acta. 2010;411(7-8):540-5

29. Prabhu SD, Frangogiannis NG. The Biological Basis for Cardiac Repair After Myocardial Infarction: From Inflammation to Fibrosis. Circ Res. 2016;119(1):91-112.

30. Frantz S, Bauersachs J, Ertl G. Post-infarct remodelling: contribution of wound healing and inflammation. Cardiovasc Res. 2009;81(3):474-81.

31. Orn S, Ueland T, Manhenke C, Sandanger O, Godang K, Yndestad A, et al. Increased interleukin-1 beta levels are associated with left ventricular hypertrophy and remodelling following acute ST segment elevation myocardial infarction treated by primary percutaneous coronary intervention. J Intern Med. 2012;272(3):267-76.

32. Bourgeois MM, Richards IS. Gender-specific differences in the urinary expression of aldosterone, IL-1 $\alpha$ and IL-1 $\beta$. Biomarkers Med. 2010;4(6):843-47.

33. Haroon J, Foureaux G, Martins A S, Ferreira A J, Reis A M, Javed Q. Gender differences in normal left ventricle of adult $\mathrm{FVB} / \mathrm{N}$ mice due to variation in interleukins and natriuretic peptides expression levels. Cytokine. 2015;71(1):54-59.

34. Lyngdoh T, Marques-Vidal P, Paccaud F, Preisig M, Waeber G, Bochud $M$, et al. Elevated serum uric acid is associated with high circulating inflammatory cytokines in the population- based CoLaus study. PLoS ONE. 2011:6(5):e19901.

35. Marques-Vidal P, Bochud M, Bastardot F, Luscher T, Ferrero F,Gaspoz J, et al. Levels and determinants of inflammatory biomarkers in a swiss populationbased sample (CoLaus study). PLoS ONE. 2011;6(6):e21002.

36. Yim J, Kim G, Lee B, Kang E S, Cha B, Kim J, et al. Relationship between circulating netrin-1 concentration, impaired fasting glucose, and newly diagnosed type 2 diabetes. Front Endocrinol. 2018;9(691). 